\title{
FACTORES DEMOGRAFICOS Y PSICOSOCIALES ASOCIADOS A LA DECISION DE CONTINUAR O TERMINAR CON EL EMBARAZO EN UN GRUPO DE ADOLESCENTES DE ESTRATO SOCIOECONOMICO BAJO
}

Silvia Becerra'

\begin{abstract}
La presente investigación tuvo como objetivo evaluar las características demográficas y psicosociales de una muestra de 60 adolescentes divididas en dos grupos de 30 sujetos cada una (adolescentes gestantes y adolescentes que han tenido un aborto inducido) y compararlas entre sí. Los resultados indicaron que existen algunas diferencias entre ambos grupos que pueden ser importantes en la decisión de aborto, como la edad y ocupación de la adolescente, su deseo de salir embarazada, el número de embarazos previos, la reacción del padre del bebé al embarazo y la satisfacción y la necesidad del soporte social en la adolescente.

Palabras Claves: adolescencia, aborto, aborto inducido, factores de riesgo.

Demographic and psychosocial factors regarding the decision to continue or interrupt a pregnancy in a group of low socioeconomic adolescents

The purpose of the present study was to assess the demographic and psycho-social characteristics of a sample of 60 adolescents divided in two groups of 30 subjects each one (pregnant adolescentes and adolescents with an induced abortion) and compare them. The results indicated the existence of some differences between both groups that may be relevant in the decision to have an abortion, for example the adolescent's age and occupation, her desire to become pregnant, the number of past pregnancies, the baby's father reaction to the pregnancy and the adolescent satisfaction and necessity of social support.

Keywords: adolescence, abortion, induced abortion, risk factors.
\end{abstract}

1. Psicóloga clínica licenciada de la Pontificia Universidad Católica del Perú. Actualmente se desempeña como docente por horas en dicha Universidad y como psicóloga de la Clínica Psiquiátrica Baltazar Caravedo. 

En la perspectiva actual del desarrollo humano se considera a la adolescencia como un período evolutivo importante, el cual tiene objetivos y tareas específicas que llevan a la persona hacia la adultez. Una de las áreas fundamentales de desarrollo en esta etapa, es la sexualidad. El desarrollo de esta área implica, desde la adquisición de una educación sexual correcta hasta el inicio de una actividad sexual responsable. Sin embargo, en ella no se dejan de suscitar problemas, y uno de los más serios es el embarazo no planificado (OMS, 1986). Cuando la adolescente se enfrenta a este hecho, tiene que tomar una decisión: continuar con el embarazo (y después quedarse con el niño o darlo en adopción) o abortar.

La decisión de hacerse un aborto y conseguirlo implica un nivel importante de tensión emocional e interpersonal, tensión que puede ser afrontada adecuadamente o llevar a conflictos psicológicos y a desajustes (Frejka, Atkin y Toro, 1987). Asimismo, está ampliamente documentado los riesgos físicos de una interrupción inducida del embarazo (Tietze, 1987). El fenómeno del aborto adquiere, así, características especiales y de particular relevancia, más aún cuando la mujer tiene entre 12 y 19 años de edad.

En los últimos años se ha dado un gran incremento en la incidencia del aborto en todo el mundo y, el Perú no escapa a esta realidad (Ferrando, Singh y Wulf, 1989). Se han llevado a cabo numerosos estudios sobre este tema, y diversas personas han opinado y opinan acerca de las formas de disminuir la incidencia del aborto inducido o de disminuir las tasas de morbilidad y mortalidad asociadas con él. Por lo tanto, en la actualidad el aborto es un tema de gran interés para todos.

Sin embargo, los estudios realizados se han desarrollado principalmente en países desarrollados, en los cuales el aborto y sus consecuencias adquieren características distintas debido a las diferencias de la población 
y contexto, y al carácter legal que éste tiene en ellos. En el Perú, se conoce muy poco acerca de los correlatos demográficos y psicológicos de la terminación del embarazo. Por ello surgió el interés y deseo de investigar y conocer sobre los diferentes aspectos de la situación de vida de las jóvenes de clase socioeconómica baja que abortan, y orientamos nuestro estudio hacia un grupo de adolescentes que acuden a un hospital a tratarse por un aborto complicado.

El objetivo de la presente investigación fue conocer las características demográficas y psicosociales que presentan un grupo de adolescentes de estrato socio-económico bajo que han terminado con su embarazo, con un especial énfasis en conocer cómo están constituidas sus redes de soporte social; y evaluar algunas diferencias que presentan con otro grupo de adolescentes, de características similares, pero que ha continuado con su gestación.

Se trata de un estudio descriptivo, en el que después de cumplido este objetivo, se procedió a comprobar una hipótesis; a saber, que existen diferencias significativas en cuanto a las características del soporte social (tamaño de redes percibidas y efectivas, satisfacción y necesidad de soporte) entre las adolescentes que continuaron con su embarazo y las que abortaron. Con las siguientes cuatro hipótesis específicas: existen diferencias significativas en cuanto al tamaño de las redes de soporte social percibido, en cuanto al tamaño de las redes de soporte social efectivas, en cuanto a la satisfacción con el soporte y en cuanto a la necesidad de soporte.

\section{Metodología}

La muestra del estudio estuvo constituida por 60 adolescentes divididas en dos grupos de 30 sujetos cada uno. Un grupo lo constituyeron adolescentes que acudieron al Instituto Materno Perinatal (Ex-Hospital Maternidad de Lima) para solicitar atención gineco-obstétrica por encontrarse embarazadas; y, el otro estuvo constituido por adolescentes que solicitaron atención y fueron atendidas en el referido Instituto, por presentar complicaciones debido a un aborto inducido. Para seleccionar la muestra, se utilizó un muestreo no probabilístico de tipo intencional, por lo que no puede considerarse a la muestra como representativa de todas las adolescentes que abortan.

54 
Debido a la dificultad para discriminar entre los abortos inducidos de aquellos que sucedieron espontáneamente según los criterios clínicos establecidos en la hoja de ingreso e historia clínica de las pacientes, se determinó que para que una adolescente sea incluida en el Grupo de Aborto, debía cumplir los siguientes requisitos:

- Aceptación por parte de la paciente de que se había sometido a un aborto, o, en su defecto:

- Tres o más de los siguientes datos clínicos:

* temperatura por encima de los $38^{\circ} \mathrm{C}$,

* frecuencia cardíaca de 100 o más pulsaciones por minuto,

* presencia de contenidos uterinos purulentos o con mal olor,

* huellas de lesión en el cuello uterino, y

* rebote peritoneal (constituye un síntoma de peritonitis).

Los datos anteriores fueron complementados con la siguiente información:

- Inconsistencias en la información dada y/o información no veraz,

- Elementos proporcionados por la entrevista, tales como:

* Embarazo no planeado y/o no deseado,

* Haber considerado en algún momento no continuar con el embarazo,

* Abandono o no apoyo por parte de la pareja,

* Falta de apoyo de la familia,

* Abortos previos, y

* Estado civil de soltera o separada.

La información para determinar los criterios de un aborto inducido fue obtenida de los informes de Belsey y Rosenfield (1989).

Los instrumentos utilizados para la presente investigación fueron:

1) Encuesta sobre el Perfil Psicosocial de la Adolescente Gestante de Estrato Socioeconómico Bajo, elaborada por Rojas (1991).

2) Encuesta sobre el Perfil Psicosocial de la Adolescente que ha Terminado con su Embarazo de Estrato Socio-Económico Bajo, basada en la encuesta anterior a la cual se adjuntó un anexo sobre el área del aborto; $y$ 
3) Inventario de Entrevista de Soporte Social de Arizona, elaborada por Barrera (1981).

Para el tratamiento de los datos, se usó la tabulación de frecuencias y medidas de tendencia central para el estudio de las características sociodemográficas de la muestra; la prueba no paramétrica para dos muestras independientes $U$ de Mann-Whitney para el contraste de las hipótesis; y medidas de odds ratio o razón de las probabilidades, el chi-cuadrado, los niveles de significación y los intervalos de confianza para evaluar los factores de riesgo del aborto.

\section{Resultados y Discusión}

En relación a los datos demográficos, se tiene que la mayoría de las adolescentes de la muestra tiene 17 o más años de edad. Aproximadamente el $60 \%$ de la muestra total ha nacido en Lima, el resto son migrantes, mayormente de la Sierra. El $50 \%$ de la muestra total vive con 6 o más personas en su casa y tienen un nivel de hacinamiento regular. Sin embargo, la mayoría cuenta con los servicios básicos de luz, agua, desagüe y baño. El 80\% de la muestra no ha terminado su secundaria o se encuentra aún estudiando. Cerca de las tres cuartas partes de las adolescentes de la muestra total ha trabajado alguna vez en su vida, pero muchas no lo hacen en la actualidad. En la muestra hay un predominio de adolescentes católicas, pero es importante resaltar que más de la mitad de ellas se catalogaron como poco religiosas. Finalmente, se observa que la mayor parte de las jóvenes de la muestra no está de acuerdo con el aborto.

En relación a la experiencia de los miembros familiares y amigos con el embarazo temprano, se observa que la mayoría de madres de las adolescentes de la muestra se embarazaron y/o tuvieron su primer hijo siendo adolescentes, más de la mitad de las adolescentes tienen amigas de su edad que tiene un hijo o están embarazadas y una tercera parte, tiene hermanas que han sido madres durante su adolescencia. Esto nos revela un alto índice de embarazo adolescente entre las personas más allegadas a la adolescente, lo cual es reportado también en otras investigaciones (OPS, s.a.; Black, 1979; Thonet, 1983). En lo que respecta a las características de la sexualidad, se observa que el promedio de la edad de inicio de la menarca es de aproximadamente 13 años. Resalta que cerca de las tres cuartas partes 56 
de las adolescentes de la muestra afirmaron tener alguna información sobre sexualidad; sin embargo, se observó que ésta era mayormente errónea e insuficiente. La principal fuente de información sobre este tema fueron los maestros de colegio. El promedio de edad de inicio de las relaciones sexuales es aproximadamente de 16 años. También resalta el alto porcentaje de adolescentes de la muestra que conoce acerca de algún método anticonceptivo. Sin embargo, un bajo porcentaje los ha usado alguna vez en su vida (30\% de adolescentes gestantes y $16.7 \%$ de adolescentes que abortaron). El método más usado ha sido el método del ritmo. También se observa que todas las adolescentes que usaron algún método anticonceptivo en el momento en que quedaron embarazadas, utilizaron este método En relación a las características del padre del bebé, se encuentra que casi todas las adolescentes de la muestra conocían al autor de su embarazo. El resumen de estos resultados se presentan en el Cuadro 1.

Resaltando en forma resumida las diferencias existentes entre ambos grupos, se aprecia, en cuanto a las características demográficas, que las adolescentes que abortaron en comparación a las gestantes son en promedio un año mayor, y que hay más cantidad de solteras y de empleadas domésticas. En relación a los aspectos psicosociales, se encuentra un mayor número eran huérfanas de padre, que no viven con su familia y que la han abandonado; además los padres y madres son mayores en 4.4 años y hay mayor cantidad de viudos y de parejas separadas. Con respecto a la pareja, autor del embarazo de la adolescente que abortó, se encuentra un mayor número que han terminado su relación con la adolescente y que no conocían de su embarazo; la reacción ante la noticia del embarazo que predomina es la "negativa" (sentimientos de preocupación, miedo, entre otros). Finalmente, en relación a la planificación y deseo de quedar embarazada, más adolescentes que abortaron no planificaron ni desearon su embarazo (ver Cuadro 2); y hay un mayor número de ellas con embarazos previos.

Entre las características diferenciales más saltantes de las adolescentes que continuaron con su embarazo, se encuentra que una mayor cantidad de ellas han dejado de trabajar, se desempeñan como ambulantes, tienen una relación actual con el padre de su bebé y la reacción principal de éste ante la noticia del embarazo fue "positiva" (sentimientos de alegría). 
Cuadro 1: Cuadro resumen de las características sobre la sexualidad de la adolescente gestante y que abortó $\left(^{*}\right)$

\begin{tabular}{|c|c|c|c|c|c|c|}
\hline \multirow[b]{2}{*}{ Características } & \multicolumn{3}{|c|}{ Gestante } & \multicolumn{3}{|c|}{ Aborto } \\
\hline & $\mathrm{F}$ & $\%$ & $\overrightarrow{\mathrm{X}}$ & $\mathrm{F}$ & $\%$ & $\overline{\mathrm{X}}$ \\
\hline $\begin{array}{l}\text { Promedio de edad de inicio } \\
\text { de la menarquía }\end{array}$ & & & 12.9 & & & 13.4 \\
\hline $\begin{array}{l}\text { Estuvieron informadas sobre } \\
\text { la menarquía }\end{array}$ & 19 & 63.3 & & 14 & 46.7 & \\
\hline Tienen información sexual & 23 & 76.7 & & 22 & 73.3 & \\
\hline $\begin{array}{l}\text { Promedio de edad de inicio } \\
\text { de las relaciones sexuales }\end{array}$ & & & 15.7 & & & 16.2 \\
\hline $\begin{array}{l}\text { Conocen a su primera pareja } \\
\text { sexual }\end{array}$ & 30 & 100. & & 29 & 96.7 & \\
\hline $\begin{array}{l}\text { Adolescentes que tienen algún } \\
\text { grado de parentesco con su } \\
\text { primera pareja sexual }\end{array}$ & 4 & 13.3 & & $1^{1}$ & 3.4 & \\
\hline $\begin{array}{l}\text { Mediana del número de parejas } \\
\text { sexuales }\end{array}$ & & & 1 & & & 1 \\
\hline $\begin{array}{l}\text { Conocen sobre métodos } \\
\text { anticonceptivos }\end{array}$ & 26 & 86. & & 27 & 90.0 & \\
\hline
\end{tabular}

$\left(^{*}\right.$ La lectura de este cuadro se realizará por filas

Nota: $\mathbf{n}=30$ para todos los casos, a excepción de ${ }^{1}$ y los porcentajes son sobre 100

${ }^{1} \mathrm{n}=29$. Número de adolescentes que conocen a su primera pareja sexual.

Cuadro 2: Cuadro resumen de las características sobre la sexualidad de la adolescente gestante y que abortó $\left(^{*}\right)$

\begin{tabular}{|c|c|c|c|c|}
\hline \multirow[b]{2}{*}{ Características } & \multicolumn{2}{|c|}{ Gestante } & \multicolumn{2}{|c|}{ Aborto } \\
\hline & F & $\%$ & $\mathrm{~F}$ & $\%$ \\
\hline No planificó su embarazo & 25 & 83.3 & 28 & 93.3 \\
\hline No desearon salir embarazadas & 20 & 66.7 & 27 & 90.0 \\
\hline
\end{tabular}

(*) La lectura de este cuadro se realizará por filas.

Nota: $n=30$ en todos los casos y los porcentajes son sobre 100

58 
En relación a la situación del aborto en las adolescentes que abortaron, se observa que el $60 \%$ reconoció tratarse de un aborto inducido. La mayor parte de ellas dio más de una razón para abortar, pero el motivo más frecuentemente mencionado fue por el miedo que experimentaron a que sus padres y/o familiares se enteraran del embarazo y se molestaran con ella. El segundo motivo más mencionado fue el deseo de terminar sus estudios (ver Cuadro 3).

Cuadro 3: Distribución de las adolescentes que abortaron de acuerdo a los motivos para abortar $\left(^{*}\right)$

\begin{tabular}{|l|rr|}
\hline \multirow{2}{*}{ Motivos } & \multicolumn{2}{|c|}{$\mathrm{N}=18$} \\
\cline { 2 - 3 } Muy joven aún & $\mathrm{F}$ & $\%$ \\
Deseo de terminar estudios & 7 & 38.9 \\
Miedo a que padres y/o familiares se enteren & 8 & 44.4 \\
Dificultades económicas & 9 & 50.0 \\
Abandono o no apoyo de pareja & 4 & 22.2 \\
Problemas de pareja & 5 & 27.8 \\
Otro hijo que mantener & 2 & 11.1 \\
Bebé interfiere con planes futuros & 1 & 5.6 \\
Sentimiento de incapacidad de criar al niño & 2 & 11.1 \\
Pareja no está preparada para ser padre & 2 & 11.1 \\
Violación & 1 & 5.6 \\
\hline
\end{tabular}

(*) La lectura de este cuadro se realizará por filas.

Nota: los porcentajes han sido calculados sólo en el grupo de adolescentes que afirmaron tratarse de un aborto inducido.

En cuanto a las características de las redes de soporte social de las adolescentes y la comprobación de la hipótesis planteada en la investigación, se aprecia que las adolescentes gestantes perciben, en promedio, que nueve personas le pueden proveer de soporte en algún momento pero que en el último mes, sólo entre siete y ocho personas se la han dado. En cambio las adolescentes que abortaron perciben en promedio a diez personas dentro de sus redes de soporte pero que solamente entre cinco y seis de ellas le han proveído de este soporte en el último mes. A pesar de observar las diferencias mencionadas entre ambos grupos, éstas no son 
estadísticamente significativas; por consiguiente, la primera y segunda hipótesis específica planteadas en la presente investigación no se cumplen, es decir, no se encontraron diferencias estadísticamente significativas en el tamaño de las redes percibidas y utilizadas por las adolescentes de ambos grupos. Sin embargo, las hipótesis específicas tres y cuatro sí se confirman: se encontró que las adolescentes que abortaron estaban menos satisfechas con el soporte recibido y habían necesitado más de éste en el último mes, en comparación a las gestantes. Esto nos indica la gran necesidad que tuvieron las adolescentes que abortaron de confiar a alguien su situación $y$, posiblemente, de pedir consejos que la orienten sobre la decisión a tomar. Sin embargo, muchas de ellas no obtuvieron la ayuda que requerían, quedándose insatisfechas.

Finalmente se quiere resaltar los factores de riesgo encontrados que incrementan la probabilidad de que ante un embarazo, una adolescente decida terminar con éste. El criterio de factor de riesgo se entiende por la presencia de ciertos factores biológicos, psicológicos, genéticos, ambientales, sociales o económicos y la interacción de ellos que hace vulnerable a un individuo de sufrir una "enfermedad" (Sotelo Figueiredo, 1993). Estos factores de riesgo se obtienen de la comparación de ciertas características relevantes de las adolescentes de ambos grupos por medio de la medida estadística del odds ratio. Así, se encontró que existe un mayor riesgo para abortar cuando la adolescente tiene una mayor edad. Asimismo, se observa que existe un riesgo para abortar:

* 3 veces mayor, en las adolescentes que se encuentran estudiando y/o trabajando que en aquellas que se dedican a las labores domésticas o no están haciendo nada por el momento;

* 4 veces mayor, cuando la reacción del padre del bebé ante la noticia del embarazo de la adolescente fue negativa, que cuando fue positiva;

* casi 5 veces mayor, cuando el embarazo no fue deseado que cuando sí lo fue; $y$

* 6 veces mayor, cuando se ha tenido embarazos previos que cuando se trata del primer embarazo. 


\section{Recomendaciones}

- Se sugiere acortar las encuestas, centrándose en los datos más relevantes que permitan establecer semejanzas y diferencias entre las adolescentes que continúan con su embarazo y las que abortan, incluyendo quizás también un grupo de adolescentes sexualmente activas pero que no están embarazadas; asimismo,

- Sería deseable ampliar el tamaño de la muestra, con el fin de evaluar más finamente los resultados encontrados.

- Se piensa que es necesario ampliar el conocimiento que se tiene de las adolescentes que abortan, sobre todo del proceso de toma de decisión. También se hace necesario identificar grupos de riesgo más proclives a abortar con el fin de diseñar intervenciones basadas principalmente en proporcionar información precisa y adecuada sobre sexualidad y facilitar un mayor acceso a la planificación familiar.

- Por último, se piensa que los programas de planificación familiar orientados a las adolescentes deben basarse necesariamente en un conocimiento de las características de desarrollo de la etapa adolescente, con el fin de que estos sean efectivos.

\section{Referencias}

Barrera, M. (1981). Social support in the adjustment of pregnant adolescents: assessment issues. En: M. Barrera, Jr.(Ed.), Social networks and social support. (pp. 69-96). Beverly Hills: Sage.

Belsey, M. y Rosenfield (1989). World Health Organization studies differentiating between spontaneous and induced abortions. En: F. Coeytaux, A. Leonard \& E. Royston (Eds.), Methodological issues in abortion research. Nueva York: The Population Council.

Black, D. (1979). Antecedent factors in teenage pregnancy. Fertility and Contraception, 3, (4) 59-64.

Chirinos, J. (1993). El aborto en el Perú: estudio epidemiológico hospitalario en las ciudades de Iquitos, Piura y Puno. Tesis para optar por el Grado de Doctor en Medicina. Lima: Universidad Peruana Cayetano Heredia. 
Coeytaux, F. (1992). El aborto inducido: una cuestión de salud pública para las Américas. Nueva York: Population Council.

Ferrando, D.; Singh, S. y Wulf, D. (1989). Adolescentes de hoy, padres del mañana. Lima: The Alan Guttmacher Institute.

Frejka, T.; Atkin, L. y Toro, O.L. (1987). Programa de investigación para la prevención del aborto inducido en condiciones riesgosas y sus consecuencias adversas en América Latina y el Caribe. México DF: The Population Council.

Organización Mundial de la Salud (OMS) (1986). Young people's health - a challenge for society. Ginebra: World Health Organization.

Organización Panamericana de la Salud (OPS) (s.a.) La salud de los adolescentes y jóvenes en las Américas: un compromiso con el futuro. Washington DC: Organización Panamericana de la Salud.

Rojas, R. (1991). Perfil psico-social de la adolescente gestante de estrato socioeconómico bajo. Memoria para optar el Grado de Bachiller en Humanidades con mención en Psicología. Lima: Pontificia Universidad Católica del Perú.

Sotelo Figuiredo, J.M. (1993). El enfoque de riesgo y la mortalidad materna: una perspectiva latinoamericana. Boletín de la Oficina Sanitaria Panamericana, 114 (4), 289-301.

Thonet, C. (1983). Embarazo en adolescentes solteras. En: G. Delgado (Ed.) (1987). Adolescencia: aspectos médicos, psicológicos y sociales. Santiago de Chile: Asociación Chilena de Protección de la Familia.

Tietze, C. (1987). Informe mundial sobre el aborto. Madrid: Ministerio de Cultura, Instituto de la Mujer. 